\title{
PRODUCCIÓN DE MADERA Y CARBONO EN LA REGENERACIÓN DE SISTEMAS AGROFORESTALES EN LA RESERVA INDÍGENA DE TALAMANCA, COSTA RICA
}

Wilson A. Calero Borge ${ }^{[21]}$

\section{Resumen}

El trabajo se desarrolló en la Reserva Indígena de Talamanca, Costa Rica. Se remidieron 64 parcelas de cacao (C) y banano (B), para estimar el almacenamiento y el incremento de madera y carbono de laurel (Cordia alliodora) y cedro amargo (Cedrela odorata L.) de regeneración natural en estos sistemas agroforestales (SAF). Se realizó inventario de laurel y cedro real y se midieron árboles con $d^{3} 4 \mathrm{~cm}$. El volumen comercial (Vc) $\left(d^{3} 45 \mathrm{~cm}\right)$ se proyectó con matrices de transición tipo Usher. Los incrementos de madera y carbono se obtuvieron en el período comprendido 2001-2005.

No hubo diferencias significativas $(\mathrm{P}<0.05)$ para la densidad $(\mathrm{d})$ y existencias totales de madera (Vt) en los SAF, la d es de 52,4 árboles ha-1 en $C$ y 53,8 árboles ha-1 en $\mathrm{B}$, contienen un volumen total de $55,4 \mathrm{~m}^{3}$ ha- ${ }^{1}$ en $C$ y $52,6 \mathrm{~m}^{3}$ ha $^{-1}$ en $B$. Las tasas de fijación de carbono son de 1,3 y 1,8 t ha $^{-1}$ año $^{-1}$ en $C$ y $B$ respectivamente.

A cinco años de proyección los C producirán $52,0\left(\mathrm{~m}^{3} \mathrm{ha}^{-1}\right)$ y los $B 54,2\left(\mathrm{~m}^{3} \mathrm{ha}^{-1}\right)$ de Vc con un IPAVc de 4,08 y 4,74 ( $\mathrm{m}^{3}$ ha $^{-1}$ año-1) en C y B respectivamente. Para todos los SAF evaluados el aprovechamiento de madera será sostenible, con las actuales tasas de aprovechamiento y reclutamiento de individuos. Se recomienda aplicar del modelo propuesto para el diseño y manejo de SAF.

Palabras claves: sistemas agroforestales, regeneración natural, inventario, matrices de transición, diámetro de corta, turnos de corta.

\section{Introducción}

Los sistemas agroforestales (SAF) son formas de uso de la tierra que se caracterizan por contener en sus diversos componentes grandes cantidades de biomasa y abundantes

[21] Máster en Agroforestería del Trópico. Coordinador de Investigación y Posgrado URACCAN Nueva Guinea.

108 | CIENCIA E INTERCULTURALIDAD, Volumen 4, Año 2, No. 2, Junio 2009 
acumulaciones de elementos como el carbono (Nair; 1997). Así mismo, el componente arbóreo maderable presente en los SAF es un recurso valioso que brinda diferentes beneficios como sombra a cultivos y ganado, madera y leña, además de prestar servicios ambientales como la captura de carbono, protección del suelo, conservación de biodiversidad, entre otros (Pezo e Ibrahim 1999; Barrance et al., 2003; Albertin y Nair 2004).

El cacao y banano son cultivos que las comunidades indígenas de Talamanca han cultivado desde antaño, bajo diferentes doseles de sombra y constituyen sus principales actividades económicas. El laurel (Cordia alliodora) y cedro real (Cedrela odorata) son las principales especies maderables en las fincas de indígenas de Talamanca. Los agricultores obtienen ingresos económicos al aprovecharlas en épocas de baja producción o bajos precios del cacao (Duguma et al., 1999; Albertin y Nair 2004) tal como sucedió cuando la moniliasis (Moniliophthora roreri) destruyó la cosecha del cacao, la madera de laurel y cedro constituyó el único ingreso de los agricultores (Beer 1980).

Los sistemas SAF tienen alto potencial para almacenar y fijar carbono en la biomasa aérea (Duguma et al., 2001; Roshetko et al., 2002; Tufekcioglu et al., 2003; Sharrow and Ismail 2004). Estudios en África señalan que los SAF con cacao pueden contener el $66 \%$ del total de biomasa del carbono que contiene un bosque primario (Duguma et al., 2001) debido a la acumulación del carbono en los árboles (Pandey 2002; Montagnini y Nair 2004) y a su rápido crecimiento y productividad (Kursten 2000). Es necesario estimar las tasas de fijación y almacenamiento de carbono de los SAF con cacao y banano manejados tradicionalmente por agricultores y productores indígenas, cuantificar económicamente su valor y asignarles un pago por el servicio ambiental prestado (Ávila 2000; Márquez 2000).

Considerando lo anterior el presente trabajo estima las existencias y los incrementos de madera y carbono en las especies de laurel (Cordia alliodora) y cedro real (Cedrela odorata L.) de regeneración natural en SAF de cacao y banano en la Reserva Indígena de Talamanca empleando un modelo de matrices de transición.

\section{Marco teórico}

\section{El laurel y cedro real en sistemas agroforestales (SAF)}

El laurel tiene características apropiadas como árbol de sombra de café (Coffea sp) o cacao (Theobroma cacao); es de fuste recto y delgado de 15-30 m, con mínima bifurcación, de copa angosta, rala, abierta y notoria capacidad de autopoda, se puede 


\section{SISTEMA AGROFORESTAL Y PRODUCCIÓN PECUARIA}

propagar por pseudoestacas (Greaves y McCarter 1990; Boshier y Lamb 1997; Calvo y Meléndez 1999). En la zona de Talamanca (Costa Rica) y Bocas del Toro (Panamá), el laurel regenera profusamente en los cacaotales, constituyéndose en una de las especies principales del dosel de sombra (Somarriba 1994; Somarriba et al., 2003).

La presencia del cedro real en SAF es un sistema tradicional de producción campesina, bajo esta forma de producción alcanza rangos altos de crecimiento en diámetro y altura. En Colombia, en asocio con frutales a los ocho años de edad tiene un incremento medio anual de $1,7 \mathrm{~m}^{2} \mathrm{n}^{-1}$ en altura y $2,6 \mathrm{~cm}$ año-1 en diámetro a la altura del pecho -dap - (Guevara 1988), mientras Ford (1979) en asocio cedro-café reporta un incremento de $2,5 \mathrm{~cm}$ año ${ }^{-1}$ en dap.

\section{Regeneración y crecimiento del laurel y cedro real en SAF}

El árbol de laurel produce grandes cantidades de semillas que son dispersadas por el viento y le permite formar rodales casi puros en áreas cercanas al árbol reproductor (CATIE 1994). Una planta adulta de laurel produce en promedio $1,3 \mathrm{~kg}$ año ${ }^{-1}$ de semillas (40000 a 100000 semillas), las cuales son dispersadas en un radio de aproximadamente 20 a 40 m (Colán 1995; Boshier y Lamb 1997). Con esta información y proponiendo un promedio (70000 semillas) y una germinación del 50\%, Somarriba (1999) estableció que un árbol reproductor potencialmente puede producir anualmente 145 plantas $\mathrm{m}^{2}$ en su vecindad inmediata. En estudios de regeneración natural de laurel en sistemas silvopastoriles se han encontrado promedios de hasta 1120 plántulas ha $^{-1}$ (Camargo et al., 2000).

Vega (1987) encontró que el cedro tuvo mayor sobrevivencia (98\%), mayor crecimiento en altura $\left(2 \mathrm{~m}_{\text {año }}{ }^{-1}\right)$ y mayor incremento diamétrico $\left(2 \mathrm{~cm}\right.$ año $\left.{ }^{-1}\right)$ en el SAF que en plantación pura. Guevara (1988) reporta que en Colombia, los mejores resultados se obtuvieron cuando el cedro amargo fue plantado en zonas de llanuras aluviales no inundables que en llanuras inundables, alcanzando hasta $3,3 \mathrm{~m}$ año-1 en altura y hasta $2,2 \mathrm{~cm}$ año ${ }^{-1}$ en dap.

\section{Modelos de crecimiento}

El crecimiento se define como el aumento en tamaño o unidad de peso (biomasa) de un organismo viviente o de una comunidad en un período de tiempo dado y su cuantificación se denomina incremento. En la producción de madera el crecimiento generalmente se relaciona al fuste o parte utilizable del árbol y se expresa en volumen (Vincent, 1980; Castillo, 1997). 


\section{Las matrices de Usher}

Las matrices de Usher intentan reducir el largo número de parámetros requeridos para formar una cadena de Markov, seleccionando el intervalo de tiempo y una amplitud de clase en la que un árbol durante ese intervalo no pueda crecer más que una clase, permite una sustancial reducción en el número de parámetros a ser estimados, entonces, la matriz puede ser reducida a cuatro vectores: crecimiento, fecundidad, cosecha y mortalidad (Vanclay 1994).

Dado que la matriz $M$ es constante, cualquier vector de distribución de edad inicial tenderá a una distribución constante (población estacionaria), donde cada clase permanece proporcionalmente constante en el tiempo con respecto a las demás pero la población total puede incrementarse, permanecer constante o decrecer. Alcanzado este punto, $M_{n}=\lambda_{n}$, donde $\lambda$ es un escalar denominado "raíz latente de la matriz $M$ ". Entonces, después de que la población se hace constante, $\lambda$ toma el lugar de $M$, esto es: $n_{t}=\lambda_{t}^{*} n_{0}$ (Vandermeer 1981).

\section{Aplicaciones de los modelos matriciales de Usher}

Diversas aplicaciones de estos modelos matriciales para árboles en sistemas agroforestales han sido desarrolladas. Somarriba (1984) empleó un modelo de matrices de transición para describir y simular la dinámica de la población de Goethalsia meiantha y facilitar la interpretación sucesional de esta especie y del bosque en su conjunto.

Para evaluar el impacto del aprovechamiento de la especie Euterpe predatoria en un bosque alto en Bolivia, se emplearon matrices de transición utilizando tasas de reclutamiento, supervivencia y crecimiento para la modelación de las poblaciones (Peña-Claros y Zuidema; 2000). Otras aplicaciones del método en bosques mixtos son las que describe Modrego et al. (2003) y Sanqueta et al. (1998) para rodales jóvenes de Pinus taeda.

\section{Importancia de los sistemas agroforestales en la fijación de carbono}

La fijación y almacenamiento de carbono en los SAF son una alternativa para dar valor agregado a la producción que podría tener un gran potencial e importancia para los productores (Ávila et al., 2001). En algunos casos, el carbono acumulado en estos sistemas es similar a aquellos encontrados en bosques secundarios, en este contexto son una opción para almacenar carbono (Montenegro y Abarca 1999). El carbono se almacena en la biomasa viviente (arriba y bajo del suelo), materia orgánica muerta (desechos y madera muerta) y en la materia orgánica del suelo (IPCC 2003). 
SISTEMA AGROFORESTAL Y PRODUCCIÓN PECUARIA

\section{Materiales y métodos}

\section{Descripción del área de estudio}

El trabajo se desarrolló en la Reserva Indígena de Talamanca, Costa Rica, la población pertenece $80 \%$ a la etnia Bribri, Cabécares $15 \%$, 3\% son afrobribris, el restante $2 \%$ tiene diversos orígenes culturales y nacionales (Borge y Castillo 1997).

\section{Población de estudio}

La población de estudio consistió en los cacaotales y bananales de Talamanca. En 20 comunidades se remidieron 64 parcelas que fueron establecidas en el año 2000, la parcela de medición correspondió a la plantación de cacao o banano que maneja el agricultor en la finca por lo que éstas fueron de tamaño y forma variable.

\section{Inventario de laurel y cedro}

Las especies principales del dosel de sombra en cacaotales y bananales de Talamanca (Guiracocha 2000) son laurel y cedro real, todos los árboles de estas especies con diámetro a la altura del pecho (dap) $\square 4 \mathrm{~cm}$ fueron medidos.

Se designó como cacaotal (C) las plantaciones puras de cacao y a las que tenían cacao combinado (siendo cacao predominante) y se designó como bananales (B) a las plantaciones puras de banano y a las que tenían banano combinado (siendo banano predominante). Se realizó una diferenciación según unidades fisiográficas de valle (V) y ladera (L).

\section{Rendimiento de madera y tasas de aprovechamiento de laurel y cedro amargo}

\section{Almacenamiento y rendimiento de madera, biomasa y carbono}

Con los datos de Dap del inventario de laurel y cedro real se definieron 17 clases diamétricas con un ancho de clase de $5 \mathrm{~cm}$. Para el cálculo de los diferentes parámetros dasométricos, biomasa y carbono, se siguió el procedimiento siguiente:

- El volumen del fuste para árboles individuales de laurel se estimó según Somarriba y Beer (1987):

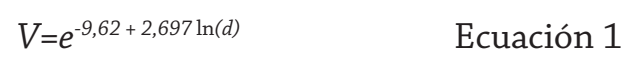


Donde:

$V$ : Volumen del fuste con corteza $\left(\mathrm{m}^{3}\right) ;$ e:Base de los logaritmos naturales; d:Diámetro a la altura del pecho $(\mathrm{cm})$.

El volumen comercial del fuste de cedro amargo en árboles individuales se estimó con la ecuación de Ford (1979) con dap $\geq 22.4 \mathrm{~cm}$.

$V_{c}=0,21245\left(d^{2}\right)-7,02212 \quad$ Ecuación 2

Donde:

$V_{c}:$ Volumen comercial con corteza (pmt); d:diámetro a la altura del pecho $(\mathrm{cm}) ; 1$ pulgada maderera tica $(\mathrm{pmt})=0.0028 \mathrm{~m}^{3}$

- Cálculo del volumen total de madera para las dos especies

$\mathrm{V}=\Sigma\left(f c d^{*} v m c\right) \quad$ Ecuación 3

Donde:

$V$ : Volumen de la especie $\left(\mathrm{m}^{3}\right) ; v m c:$ Volumen en la marca de clase $\left(\mathrm{m}^{3}\right) ; f c d$ Frecuencia de clase diamétrica.

- Calculo del área basal de las especies de cedro amargo y laurel en los SAF evaluados se empleó la siguiente ecuación:

$B A=1 / 4 \Pi^{*}$ dap $\quad$ Ecuación 4

Donde:

BA: Área basal $\left(\mathrm{m}^{2}\right)$; dap: Diámetro a la altura del pecho (m)

- Calculo de la biomasa de laurel y cedro real.

$B_{t}=V^{*} g^{*} f e b \quad$ Ecuación 5

Donde:

B: Biomasa total arriba del suelo (kg); V: Volumen del árbol $\left(\mathrm{m}^{3}\right)$; feb: Factor de expansión de la biomasa. 


\section{SISTEMA AGROFORESTAL Y PRODUCCIÓN PECUARIA}

- El carbono se estimó asumiendo un valor de contenido de 0,46 según (Segura 2005). Para cedro real se asumió una fracción 0,50 (IPCC 2003).

$C=B^{*} 0,46 \quad$ Ecuación 6

Donde:

C: Carbono (kg); B: Biomasa (kg).

- Los incrementos anuales de biomasa y carbono fueron calculados en base a los inventarios realizados por Suárez (2001) y el de este estudio (2005).

\section{Modelación de la dinámica poblacional de laurel en sistemas agroforestales de Talamanca}

Se proyectó la dinámica poblacional desarrollando un modelo de matrices de transición tipo Usher que proponen Somarriba (2001) y Suárez (2001). La población fue dividida en clases diamétricas de $5 \mathrm{~cm}$, tal como el usado en estudios semejantes (Somarriba, 1990; Suárez, 2001). El diámetro mínimo de corta fue establecido en $45 \mathrm{~cm}$ y es el más utilizado por los agricultores de Talamanca.

La iteración se realizó por períodos de un año hasta una proyección de cinco años para determinar la capacidad de producción de madera y comportamiento de la población de laurel de los SAF en estudio. La ecuación matricial empleada fue la siguiente:

$$
n_{t+1}=M^{*} n_{t}+\mathrm{R} \quad \text { Ecuación } 7
$$

Donde los vectores $n_{t+1}$ y $n_{t}$ denotan la distribución diamétrica (árboles ha ${ }^{-1}$ ) entre los años sucesivos $t$ y $t+1, M$ es la matriz de coeficientes de transición y $R$ es el reclutamiento de individuos en el período que se analiza. La ecuación $n_{t+1}=M^{*} n_{t}+\mathrm{R}$ devuelve en cada iteración en la clase diamétrica superior la densidad de árboles que alcanzan el diámetro mínimo de corta.

Suponiendo una población de $z$ clases diamétricas $\left(z_{1}, z_{2}, z_{t}\right)$ en el año $t$, en donde $z_{\mathrm{t}}$ es la clase diamétrica de los árboles de cosecha, la proyección de la población en el año $t+1$ con la ecuación $n_{t+1}=M^{*} n_{t}+\mathrm{R}$ en forma matricial es: 


$$
\left|\begin{array}{c}
a \\
z_{1, t+1} \\
z_{1, t+1} \\
- \\
- \\
z_{1, t+1}
\end{array}\right|=\left|\begin{array}{cccccc}
1 & 0 & 0 & 0 & 0 & 0 \\
e_{i j} & r_{i i} & 0 & 0 & 0 & 0 \\
0 & e_{i j} & r_{i i} & 0 & 0 & 0 \\
0 & 0 & - & - & 0 & 0 \\
0 & 0 & 0 & - & - & 0 \\
0 & 0 & 0 & 0 & e_{i j} & r_{i i}
\end{array}\right| *\left|\begin{array}{c}
a \\
z_{1, t} \\
z_{1, t} \\
- \\
- \\
z_{i, t+1}
\end{array}\right|+R
$$

Donde:

$-e_{j}$ : Coeficientes de transición del período $i$ al período $j$

$$
e_{j}=p_{j}^{*} S_{i} \quad \text { Ecuación } 8
$$

$-r_{i i}$ : Coeficientes de permanencia en el período $i$

$$
r_{i i}=q_{i}^{*} S_{i} \quad \text { Ecuación } 9
$$

El cálculo de estos coeficientes fue derivado de la estimación de una tasa anual de paso $\left(P_{i j}\right)$ de clase $i$ a clase $j$ y una tasa anual de permanencia $\left(q_{i i}\right)$ en la misma clase $i$ ajustados por un factor de supervivencia anual por clase $\left(S_{i}\right)$, entonces:

$$
P i j=i_{d} / K_{i} \quad \text { Ecuación } 10
$$

- Donde $i_{d}$ denota el incremento corriente anual en diámetro en la clase $i$ estimado con la primera derivada de la ecuación de crecimiento de Chapman Richards (Suárez 2001) y $K_{i}$ el intervalo de clase $(5 \mathrm{~cm})$.

$$
i_{d}=a^{*} b^{*} c^{*} e^{-b}\left(1-e^{-b}\right)^{c-1} \quad \text { Ecuación } 11
$$

\section{Donde:}

$a$; $b$; $c$ : Parámetros de la ecuación de regresión; e: Base de los logaritmos; $t$ : Edad para alcanzar la categoría diamétrica

- Entonces la tasa anual de permanencia se calculó de la siguiente manera:

$q_{i i}=1-p_{j} \quad$ Ecuación 12

$q_{i i}:$ Tasa anual de permanencia 


\section{SISTEMA AGROFORESTAL Y PRODUCCIÓN PECUARIA}

$p_{i j}$ : Tasa anual de paso de la clase $i$ a la clase $j$

La supervivencia anual por clase $\left(S_{i}\right)$ se estimó con base en la distribución diamétrica del inventario del 2005 y las tasas de aprovechamiento anual por clase diamétrica para laurel tomando en cuenta que durante el inventario actual no se reportaron individuos muertos por otra razón diferente al aprovechamiento. La ecuación que se empleó fue la siguiente:

$$
S_{i}=\left(N_{i}-T_{i}\right) / N_{i} \quad \text { Ecuación } 13
$$

Donde $S_{i}$ es la supervivencia en la clase $i ; N_{i}$ es el número de individuos en la clase diamétrica $i$ y $T_{i}$ es la tasa de aprovechamiento anual para la clase diamétrica $i$.

El reclutamiento anual se estimó con base en la comparación de las curvas de distribución diamétrica de los inventarios del 2001 y 2005. La diferencia entre ambas curvas permitió obtener el número de individuos que se incorporaron, la ecuación empleada fue la siguiente:

$$
R i=\left(N_{t 2005}-N_{t 2001}\right) / 5 \quad \text { Ecuación } 14
$$

Donde:

$\underline{R}_{i}$ : Tasa anual de reclutamiento (árboles ha ${ }^{-1} \mathrm{año}^{-1}$ ); $N_{t 2005}$ Individuos en la clase diamétrica $i$ por hectárea en el inventario del 2005; $N_{t 2001}$ Individuos en la clase diamétrica $i$ por hectárea en el inventario del 2001.

El análisis de la dinámica poblacional de laurel se realizó a dos niveles: a nivel de poblaciones de $S A F$ cacao en valle $(\mathrm{CV})$, cacao en ladera (CL), banano en valle $(\mathrm{BV})$ y banano en ladera (BL) y a nivel de parcelas individuales.

Para evaluar el efecto en la dinámica poblacional del laurel como resultado de cambios en las prácticas de manejo, se simuló para cinco años el comportamiento de la población con diferentes tasas de sobrevivencia y reclutamiento. Los escenarios fueron: 1) proyección a cinco años con los datos observados de la distribución diamétrica (árboles $\mathrm{ha}^{-1}$ ), 2) $100 \%$ de sobrevivencia en las clases diamétricas inferiores (dap < $45 \mathrm{~cm}$ ) y 3 ) incrementos del $50 \%$ en las tasas de reclutamiento.

La proyección del rendimiento de madera se realizó a partir de la distribución poblacional en clases diamétricas, con la Ecuación 1 se estimó la producción de madera $\left(\mathrm{m}^{3} \mathrm{ha}^{-1}\right.$ año $\left.{ }^{-1}\right)$ para cada sistema agroforestal. 
Se asumió lo siguiente (para la población): si el incremento corriente anual del volumen comercial (ICAVc) es mayor que cero el aprovechamiento es sostenible, si es igual a cero está en equilibrio con el crecimiento de la población, si el ICAVc es menor que cero el aprovechamiento no es sostenible. También se determinó el incremento periódico anual del volumen comercial IPAVc. Las ecuaciones fueron:

$$
I C A V c=V c_{i+1}-V c_{i} \quad \text { Ecuación } 15
$$

Donde:

ICAVc : Incremento corriente anual del volumen comercial $\left(\mathrm{m}^{3} \mathrm{ha}^{-1} \mathrm{año}^{-1}\right) ; V c$ ${ }_{i+1}:$ Volumen comercial proyectado en el año siguiente $\left(\mathrm{m}^{3} \mathrm{ha}^{-1}\right.$ año $\left.\mathrm{o}^{-1}\right) ; V c_{i}:$ Volumen comercial proyectado en el año actual $\left(\mathrm{m}^{3} \mathrm{ha}^{-1} \mathrm{año}^{-1}\right)$

$$
I P A V c=\left(V c_{f}-V c_{i}\right) / 5 \text { Ecuación } 16
$$

Donde:

IPAVc : Incremento periódico anual del volumen comercial proyectado $\left(\mathrm{m}^{3} \mathrm{ha}^{-1}\right.$ año-1); $V c_{f}$ :Volumen comercial $\left(\mathrm{m}^{3} \mathrm{ha}^{-1}\right)$ al final del período proyectado de cinco años; $\mathrm{Vc}_{\mathrm{i}}$ : Volumen comercial al inicio del período proyectado $\left(\mathrm{m}^{3} \mathrm{ha}^{-1}\right)$.

El análisis a nivel de parcelas se realizó de la siguiente manera: las tasas de sobrevivencia y reclutamiento anual fueron calculadas mediante las Ecuaciones 13 y 14 respectivamente, la distribución diamétrica fue la encontrada en cada parcela en este estudio.

Se realizaron iteraciones para cinco años y se encontraron los incrementos en densidad (Ecuación 17) si el incremento fue positivo se consideró que hay crecimiento de la población, si es negativo entonces hay disminución de la población, se encontraron las tasas anuales de incremento considerando el período de proyección (Ecuación 18).

$$
\Delta N=N_{2010}-N_{2005} \quad \text { Ecuación } 17
$$

Dónde:

$\Delta N$ : Cambio en la densidad de árboles en el período 2005-2010(árboles ha ${ }^{-1}$ ); $N_{2010}$ : Densidad de árboles a cinco años de proyección (árboles ha ${ }^{-1}$ ); $N_{2005}$ : Inventario base en la parcela (árboles ha-1) 


\section{SISTEMA AGROFORESTAL Y PRODUCCIÓN PECUARIA}

\section{IPAN $=\Delta N / 5$ años Ecuación 18}

\section{Dónde:}

IPAN: Incrementos periódicos anuales de la densidad (árboles ha ${ }^{-1} \mathrm{año}^{-1}$ ); $\Delta \mathrm{N}$ : Cambios en la densidad de árboles en el período 2005-2010(árboles ha ${ }^{-1}$ ).

\section{Resultados y discusión}

\section{Existencias de madera de laurel y cedro amargo en sistemas agroforestales de Talamanca}

La especie laurel es la más abundante en relación al cedro amargo en los sistemas agroforestales de cacao y banano en Talamanca (Cuadro 1). No hubo diferencias significativas en las variables dasométricas evaluadas. Sin embargo, las densidades de laurel en valle tienen un área basal mayor de un $22 \%$ que las laderas. En sentido general los SAF de cacaotales en valle presentan las mayores existencias de madera, biomasa y carbono de laurel.

Las densidades de cedro amargo son bajas en relación a las presentadas por el laurel en los $S A F$ de $C$ y $B$, en relieve de $V$ y $L$. No hubo diferencias entre las diferentes variables dasométricas evaluadas. Sin embargo, el cedro real en B almacena un $26 \%$ de carbono más que los $C$, siendo estas de 1,5 y 1,4 tha $^{-1}$ para $C$ y $B$ respectivamente.

Cuando se compararon las densidades de cedro por condición de crecimiento $\mathrm{Vy}$ $\mathrm{L}$, no hubo diferencias entre las mismas; sin embargo, las densidades de cedro en $\mathrm{V}$ presentan un área basal mayor en un $20 \%$ que las densidades de cedro en L.

Cuadro 1: Existencias de laurel y cedro amargo en los SAF de cacao y banano en condiciones de ladera y valle en Talamanca, Costa Rica, en el año 2005.

\begin{tabular}{lcccc}
\hline \multicolumn{1}{c}{ Cultivo principal } & \multicolumn{2}{c}{ Cacao } & \multicolumn{2}{c}{ Banano } \\
\hline \multicolumn{1}{c}{ Unidad fisiográfica } & Valle & Ladera & Valle & Ladera \\
\hline Variable & Laurel & & & \\
\hline $\begin{array}{l}\text { densidad (árboles ha-1 dap } \\
\geq 4 \mathrm{~cm})\end{array}$ & $55,77 \mathrm{a} \pm 7,0$ & $49,11 \mathrm{a} \pm 6,7$ & $53,91 \mathrm{a} \pm 14,8$ & $53,76 \mathrm{a} \pm 16,5$ \\
\hline Área basal $\geq 4 \mathrm{~cm}\left(\mathrm{~m}^{2}\right.$ ha- 1$)$ & $5,59 \mathrm{a} \pm 0,8$ & $3,87 \mathrm{a} \pm 0,4$ & $4,604 \mathrm{a} \pm 1,2$ & $4,18 \mathrm{a} \pm 1,2$ \\
\hline
\end{tabular}


Producción de madera y carbono en la regeneración de sistemas agroforestales en la Reserva Indígena de Talamanca, Costa Rica

\begin{tabular}{|c|c|c|c|c|}
\hline \multirow{2}{*}{$\begin{array}{l}\text { Cultivo principal } \\
\text { Unidad fisiográfica }\end{array}$} & \multicolumn{2}{|c|}{ Cacao } & \multicolumn{2}{|c|}{ Banano } \\
\hline & Valle & Ladera & Valle & Ladera \\
\hline$V t \geq 4 \mathrm{~cm}\left(\mathrm{~m}^{3}\right.$ ha- 1$)$ & $66,76 a \pm 10,1$ & $44,10 a \pm 6,6$ & $56,02 a \pm 17,1$ & $49,23 a \pm 16,4$ \\
\hline$V_{c} \geq 45 \mathrm{~cm}$ (m ${ }^{3}$ ha- 1$)$ & $38,52 a \pm 7,6$ & $23,56 a \pm 6,5$ & $37,32 \mathrm{a} \pm 15,1$ & $25,06 a \pm 12,0$ \\
\hline Biomasa (t ha-1) & $49,70 a \pm 7,0$ & $35,50 a \pm 4,9$ & $40,20 \mathrm{a} \pm 11,8$ & $35,90 a \pm 11,4$ \\
\hline \multirow[t]{2}{*}{ Carbono (t ha-1) } & $23,40 a \pm 3,3$ & $16,88 a \pm 2,3$ & $18,90 a \pm 5,5$ & $16,87 a \pm 5,3$ \\
\hline & Cedro real & & & \\
\hline densidad $\geq 4 \mathrm{~cm}$ (árboles ha- 1 ) & $3,03 a \pm 1,4$ & $3,39 a \pm 1,9$ & $4,49 a \pm 1,3$ & $5,54 a \pm 3,2$ \\
\hline Área basal $\geq 4 \mathrm{~cm}$ ( $\mathrm{m}^{2}$ ha- 1 ) & $0,38 a \pm 0,1$ & $0,29 a \pm 0,1$ & $0,46 a \pm 0,1$ & $0,40 a \pm 0,2$ \\
\hline$V c \geq 45 \mathrm{~cm}\left(\mathrm{~m}^{3} \mathrm{ha}^{-1}\right)$ & $2,07 a \pm 0,9$ & $0,96 a \pm 0,5$ & $2,07 a \pm 0,9$ & $1,77 a \pm 1,7$ \\
\hline Biomasa $\left(\mathrm{t} \mathrm{ha}^{-1}\right)$ & $3,00 a \pm 1,2$ & $2,10 a \pm 0,8$ & $3,50 a \pm 1,3$ & $2,80 a \pm 1,9$ \\
\hline Carbono (t ha-1) & $1,30 a \pm 0,5$ & $1,00 \mathrm{a} \pm 0,4$ & $1,60 a \pm 0,6$ & $1,30 \mathrm{a} \pm 0,8$ \\
\hline
\end{tabular}

CV: cacao en valle; CL: cacao en ladera; BV: banano valle; BL; banano ladera; \pm : indica el error estándar; Vt: volumen total; Vc: volumen comercial; Letras iguales no difieren estadísticamente $(p>0,05)$ entre tratamientos.

La densidad del laurel es superior a las densidades reportadas por Suárez (2001) en SAF de Talamanca y por Pastrana et al. (1998) en fincas cacaoteras y bananeras de indígenas Ngöbe de Panamá. A la vez Escobar (1979), Rosero y Gewald (1979), Beer et al. (1981), Alavez et al. (1984), Somarriba y Beer (1987) y Camargo (1999) reportan densidades superiores en diferentes SAF en un rango de 78 a 305 árboles $\mathrm{ha}^{-1}$. Las densidades de cedro real son superiores a las obtenidas por Suárez (2001) en SAF de Talamanca quien reportó 1,6 y 3,0 árboles ha ${ }^{-1}$ en cacaotales y bananales respectivamente, aunque continúan siendo muy bajas.

Las bajas densidades del laurel y cedro amargo (Cuadro 1) puede deberse a que los indígenas manejan un dosel de sombra muy diversificado por lo que el sistema alberga una densidad mayor de árboles, arbustos, palmas, entre otros para satisfacer las necesidades de sombra de los cultivos.

La densidad está relacionada con el manejo de los cacaotales y bananales como son las chapias frecuentes (de 1-6 veces en cacaotales y hasta 12 veces en bananales por año) lo que provoca alta mortalidad de brinzales; al raleo de los latizales jóvenes, para regular sombra necesaria al cacao o banano y al aprovechamiento considerable de árboles con diámetros inferiores al mínimo de corta para satisfacer necesida- 


\section{SISTEMA AGROFORESTAL Y PRODUCCIÓN PECUARIA}

des de madera de pequeño porte en las viviendas, y a un incremento en la tasa de aprovechamiento de árboles aserrables.

La cantidad de carbono almacenada en el laurel y cedro real en los sistemas agroforestales de Talamanca es similar a diversos estudios. Kursten y Burschel (1993) reportan de $3-25$ t $C$ ha $^{-1}$ para árboles de sombra en SAF a densidades mayores; Alpizar et al. (1985) reportan valores de carbono en el componente arbóreo de 14,1 $\mathrm{t}$ ha $\mathrm{a}^{-1}$ para laurel en sistemas agroforestales con café en Turrialba, Costa Rica. Parece ser que las bajas densidades de laurel en los SAF de Talamanca determinan estos valores de carbono.

\section{Incrementos de madera, carbono y biomasa de laurel y cedro amargo en sistemas agroforestales de Talamanca}

El laurel alcanzó mayores incrementos que el cedro amargo en el período 2001-2005 en los SAF de $C$ y B evaluados (Cuadro 2). No hubo diferencias en los incrementos de las diferente variables dasométricas evaluadas. Sin embargo los B tienen una tasa de fijación de carbono superior en un 33\% a las tasas de fijación de los $C$, siendo estas de 1,3 y 1,8 t c ha $^{-1}$ año ${ }^{-1}$ para $C$ y $B$ respectivamente. En sentido general los SAF de $\mathrm{B}$ en $\mathrm{V}$ presentan los mayores incrementos de madera, biomasa y carbono de laurel.

Los incrementos de las densidades de cedro real son bajas en relación a los incrementos presentados por el laurel. No hubo diferencias entre los incrementos de las variables dasométricas. Sin embargo, el cedro real en $\mathrm{B}$ tiene una tasa de fijación de carbono superior a la de los $C$, siendo estas de 0,1 y $0,2 \mathrm{t} \mathrm{c} \mathrm{ha}^{-1}$ año ${ }^{-1}$ para $C$ y $B$ respectivamente.

Cuadro 2: Incrementos de laurel y cedro amargo en los SAF de cacao y banano en laderas y valles de Talamanca en el período 2001-2005

\begin{tabular}{lllll}
\hline \multicolumn{1}{c}{ Cultivo principal } & \multicolumn{2}{c}{ Cacao } & \multicolumn{2}{c}{ Banano } \\
\hline \multicolumn{1}{c}{ Unidad fisiográfica } & Valle & Ladera & Valle & Ladera \\
\hline Variables & Laurel & & & \\
\hline densidad $\geq 4 \mathrm{~cm}\left(\right.$ árboles ha $\left.^{-1} \mathrm{año}^{-1}\right)$ & $1,90 \mathrm{a} \pm 1,2$ & $1,20 \mathrm{a} \pm 0,7$ & $3,10 \mathrm{a} \pm 2,1$ & $2,40 \mathrm{a} \pm 1,5$ \\
\hline Área basal $\geq 4 \mathrm{~cm}\left(\mathrm{~m}^{2} \mathrm{ha}^{-1} \mathrm{año}^{-1}\right)$ & $0,30 \mathrm{a} \pm 0,1$ & $0,20 \mathrm{a} \pm 0,1$ & $0,40 \mathrm{a} \pm 0,1$ & $0,30 \mathrm{a} \pm 0,1$ \\
\hline $\mathrm{Vt}\left(\mathrm{m}^{3} \mathrm{ha}^{-1} \mathrm{año}^{-1}\right)$ & $3,80 \mathrm{a} \pm 1,7$ & $2,60 \mathrm{a} \pm 1,5$ & $5,80 \mathrm{a} \pm 2,2$ & $4,80 \mathrm{a} \pm 2,5$ \\
\hline $\mathrm{Vc}\left(\mathrm{m}^{3} \mathrm{ha}^{-1} \mathrm{año}^{-1}\right)$ & $2,10 \mathrm{a} \pm 1,5$ & $1,80 \mathrm{a} \pm 1,3$ & $4,60 \mathrm{a} \pm 2,2$ & $2,50 \mathrm{a} \pm 2,0$ \\
\hline
\end{tabular}




\begin{tabular}{|c|c|c|c|c|}
\hline \multirow{2}{*}{$\begin{array}{l}\text { Cultivo principal } \\
\text { Unidad fisiográfica }\end{array}$} & \multicolumn{2}{|c|}{ Cacao } & \multicolumn{2}{|c|}{ Banano } \\
\hline & Valle & Ladera & Valle & Ladera \\
\hline Biomasa (t ha-1 año-1) & $3,10 a \pm 0,7$ & $2,70 a \pm 0,6$ & $4,20 a \pm 0,7$ & $3,60 a \pm 0,7$ \\
\hline Carbono (t ha-1 año-1) & $1,50 a \pm 0,8$ & $1,20 a \pm 0,5$ & $1,90 a \pm 0,5$ & $1,70 a \pm 0,6$ \\
\hline \multicolumn{5}{|c|}{ Cedro amargo } \\
\hline $\begin{array}{l}\text { densidad } \geq 4 \mathrm{~cm} \text { (árboles ha-1 } \\
\text { año-1) }^{-1}\end{array}$ & $0,37 a \pm 0,3$ & $0,40 a \pm 0,2$ & $0,02 a \pm 0,3$ & $0,10 a \pm 0,7$ \\
\hline Área basal $\left(\mathrm{m}^{2} \mathrm{ha}^{-1}\right.$ año $\left.{ }^{-1}\right)$ & $0,04 a \pm 0,03$ & $0,04 a \pm 0,02$ & $0,07 a \pm 0,03$ & $0,07 a \pm 0,06$ \\
\hline Vc $\left(m^{3} h a^{-1} a_{n} o^{-1}\right)$ & $0,20 a \pm 0,1$ & $0,10 a \pm 0,05$ & $0,30 a \pm 0,1$ & $0,30 a \pm 0,3$ \\
\hline Biomasa (t ha-1 año-1) & $0,20 a \pm 0,2$ & $0,20 a \pm 0,1$ & $0,50 a \pm 0,2$ & $0,50 a \pm 0,4$ \\
\hline Carbono (t ha-1 año-1) & $0,10 \mathrm{a} \pm 0,1$ & $0,10 a \pm 0,05$ & $0,20 \mathrm{a} \pm 0,1$ & $0,20 \mathrm{a} \pm 0,1$ \\
\hline
\end{tabular}

\pm : indica el error estándar; $V c$ : volumen comercial; $V t$ : volumen total; Letras iguales no difieren estadísticamente $(p>0,05)$ entre tratamientos

Las tasas de fijación de carbono en los sistemas agroforestales de Talamanca 1,4 y 2,0 t C ha ${ }^{-1}$ año-1 en cacaotales y bananales respectivamente son superiores a las reportadas por Segura et al. (2005) en laurel de regeneración natural en Talamanca las que oscilaron de 0,6 a 0,8 t $C$ ha $^{-1}$ año-1. Koskela et al. (2000) en cacaotales bajo sombra de laurel y de poró en Turrialba, Costa Rica, encontraron una tasa de fijación de 4,28 t $C$ ha año ${ }^{-1}$ para el sistema cacao laurel y 3,08 t $C$ ha ${ }^{-1}$ año ${ }^{-1}$ en el sistema cacao-poró las cuales son superiores a las de este estudio.

\section{Proyección de la densidad, rendimiento e incremento del volumen comercial de madera de laurel en los sistemas agroforestales de Talamanca}

Utilizando el modelo propuesto se realizaron las proyecciones de la densidad poblacional, rendimiento e incrementos de madera. Las tasas de aprovechamiento se consideraron como las únicas fuentes de disminución de la población de laurel y fueron de 0,52 y 0,26 árboles $\mathrm{ha}^{-1}$ año-1 para los cacaotales y bananales respectivamente; el reclutamiento fue de 3,26 y 1,80 árboles $\mathrm{ha}^{-1}$ año-1 para bananales y cacaotales respectivamente, en general los bananales tienen los mejores resultados en estas dos variables, puesto que presenta las menores tasas de aprovechamiento ( $50 \%$ respecto a los cacaotales) y las mayores tasas de reclutamiento (55\% mayor con respecto a los cacaotales).

Bajo las actuales tasas de aprovechamiento y reclutamiento anual en las poblaciones de laurel y para una proyección de cinco años la densidad se incrementará en 


\section{SISTEMA AGROFORESTAL Y PRODUCCIÓN PECUARIA}

todos los sistemas agroforestales evaluados ocurriendo los mayores incrementos en los bananales, esto puede deberse a las menores tasas de aprovechamiento que ocurren en estos sistemas y a las tasas de reclutamiento más altas (Figura 1).

En todas las poblaciones la curva se desplazará hacia la derecha haciendo moda en diámetros de 30 a $35 \mathrm{~cm}$ (Figura 1), lo que indica que no se producirán incrementos considerables en las clases diamétricas inferiores, esto puede provocar una distribución poblacional donde las clases diamétricas inferiores tengan muy pocos individuos para mantener una adecuada estructura de individuos jóvenes y diámetros aprovechables.

b)
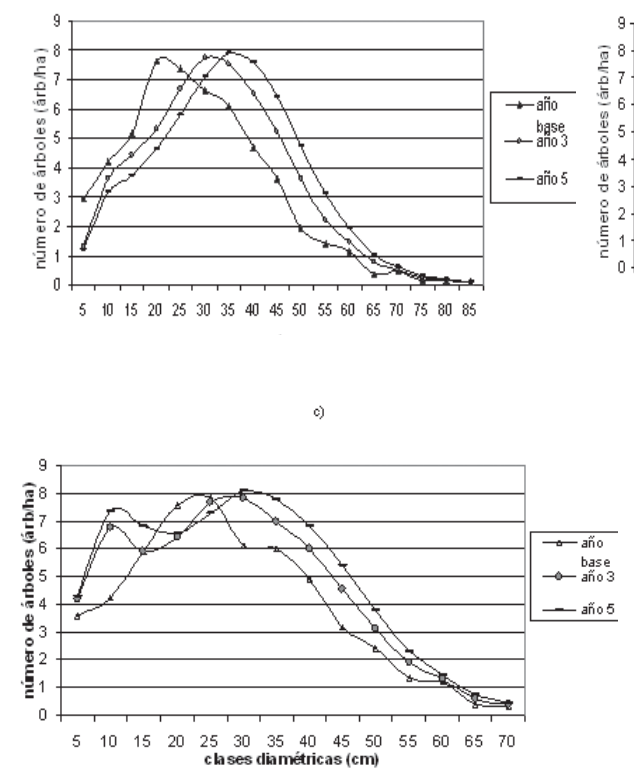

a)

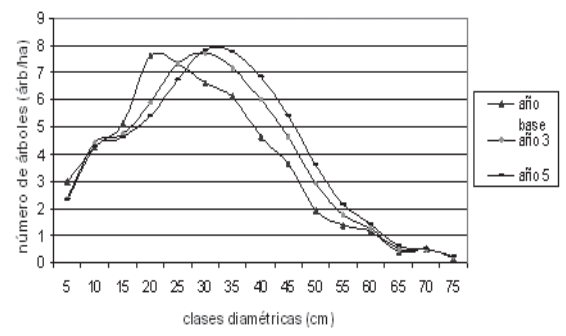

d)

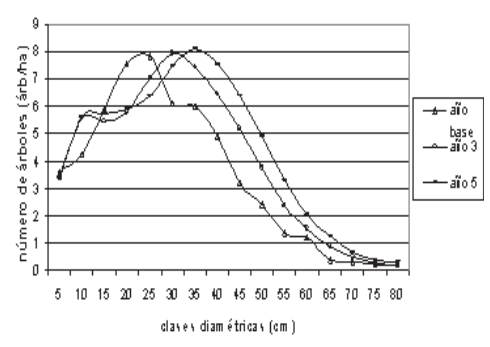

Figura 1: Proyección de la población de laurel para cinco años en a) cacao ladera, b) cacao valle, c) banano ladera y d) banano valle en Talamanca, Costa Rica.

La distribución de la población de laurel con datos observados muestra que la clase diamétrica de $20-25 \mathrm{~cm}$ tiene la mayor densidad de individuos y luego desciende hacia los diámetros superiores, siendo esto una característica de las poblaciones de regeneración natural.

La poca presencia de individuos en las clases diamétricas inferiores a $20 \mathrm{~cm}$, puede deberse a factores ecológicos como de manejo, los factores ecológicos estarían asociados a: la biomasa en descomposición sobre el suelo la cual incide en la viabilidad 
de la semilla; la sombra del dosel de árboles y del cacao y el período de lluvias puesto que el laurel germina en época seca y estas arrastraran muchas semillas. Los factores de manejo están asociados a: las frecuencia de las chapias que realiza el agricultor en la cual son eliminadas muchas plantitas; al raleo para regular sombra para el cacao o banano y que implica eliminar árboles pequeños, a partir de dap $>12 \mathrm{~cm}$ este raleo disminuye. En la proyección de la población de laurel para cinco años se observa la misma tendencia e incluso la curva hace moda en $25 \mathrm{~cm}$.

La proyección del rendimiento de madera en volumen comercial a nivel de poblaciones fue creciente en todos los sistemas agroforestales, los mayores rendimientos se producirán en los sistemas agroforestales en valle, a cinco años de proyección los SAF en laderas estarán produciendo un $30 \%$ de madera menos en relación a los SAF en valle (Cuadro 8).

Cuadro 3: Proyección de las existencias $\left(m^{3}\right.$ ha-1) de madera comercial de laurel por cinco años en cacaotales y bananales en valles y laderas de Talamanca, Costa Rica.

\begin{tabular}{lllllll}
\hline SAF & Año 0 & Año 1 & Año 2 & Año 3 & Año 4 & Año 5 \\
\hline & $\left(\mathrm{m}^{3} h \mathrm{~h}^{-1}\right)$ & $\left(\mathrm{m}^{3} \mathrm{ha}^{-1}\right)$ & $\left(\mathrm{m}^{3} \mathrm{ha}^{-1}\right)$ & $\left(\mathrm{m}^{3} \mathrm{ha}^{-1}\right)$ & $\left(\mathrm{m}^{3} \mathrm{ha}^{-1}\right)$ & $\left(\mathrm{m}^{3} \mathrm{ha}^{-1}\right)$ \\
\hline $\mathrm{CL}$ & 30,40 & 33,80 & 35,09 & 37,96 & 41,02 & 44,31 \\
\hline $\mathrm{CV}$ & 33,11 & 34,89 & 40,15 & 46,07 & 52,64 & 59,85 \\
\hline $\mathrm{BL}$ & 28,70 & 31,34 & 34,39 & 37,59 & 41,14 & 44,86 \\
\hline $\mathrm{BV}$ & 32,80 & 35,52 & 41,50 & 48,15 & 55,50 & 63,65 \\
\hline
\end{tabular}

CV: cacao en valle; CL: cacao en ladera; BV: banano valle; BL; banano ladera; SAF: sistemas agroforestales; año: reflejan el volumen comercial $\left(\mathrm{m}^{3} \mathrm{ha}^{-1}\right.$ año $\left.{ }^{-1}\right)$ en el año de proyección.

El ICAVc fue creciente en todos los sistemas agroforestales a excepción del cacao en ladera que disminuyó en el tiempo, los BV fueron los que presentaron los mayores ICAVc. El incremento periódico anual del volumen comercial (IPAvc) será mayor en los sistemas agroforestales en valle (Cuadro 9). A nivel de población en cacaotales y bananales el aprovechamiento es sostenible puesto que hay incrementos en la producción de madera. 


\section{SISTEMA AGROFORESTAL Y PRODUCCIÓN PECUARIA}

Cuadro 4: Proyección de los incrementos volumétricos de madera comercial (dap $\geq 45 \mathrm{~cm}$ ) de laurel por cinco años en cacaotales y bananales en valles y laderas de Talamanca, Costa Rica.

\begin{tabular}{lllllll}
\hline SAF & $\begin{array}{r}\text { ICAVc } \\
\text { año 1 }\end{array}$ & $\begin{array}{r}\text { ICAVc } \\
\text { año 2 }\end{array}$ & $\begin{array}{c}\text { ICAVc } \\
\text { año 3 }\end{array}$ & $\begin{array}{r}\text { ICAVc } \\
\text { año 4 }\end{array}$ & $\begin{array}{r}\text { ICAVc } \\
\text { año 5 }\end{array}$ & IPAvc \\
\hline $\mathrm{CL}$ & 3,40 & 1,29 & 2,87 & 3,06 & 3,29 & 2,78 \\
\hline $\mathrm{CV}$ & 1,78 & 5,26 & 5,92 & 6,58 & 7,21 & 5,38 \\
\hline $\mathrm{BL}$ & 2,64 & 3,05 & 3,20 & 3,55 & 3,72 & 3,23 \\
\hline $\mathrm{BV}$ & 3,14 & 5,98 & 6,65 & 7,35 & 8,15 & 6,25 \\
\hline
\end{tabular}

CV: cacao en valle; CL: cacao en ladera; BV: banano valle; BL; banano ladera; SAF: sistemas agroforestales; ICAVc: incremento corriente anual en volumen comercial $\left(\mathrm{m}^{3}\right.$ $\mathrm{ha}^{-1}$ año $\left.{ }^{-1}\right)$; IPAVc: incremento periódico anual del volumen comercial $\left(\mathrm{m}^{3} \mathrm{ha}^{-1} \mathrm{año}^{-1}\right.$ )

La densidad de laurel disminuirá en el 40 y $50 \%$ de las parcelas de banano y cacao respectivamente. Mientras que el 14 y el $17 \%$ de las parcelas de banano y cacao respectivamente tendrán altos incrementos de densidad; este resultado sugiere que el incremento de la densidad que se observa a nivel de poblaciones de SAF es producto del alto incremento de pocas parcelas en los sistemas agroforestales evaluados, mientras que en la mayoría de las parcelas la densidad está disminuyendo o tienen incrementos bajos (Figura 2).

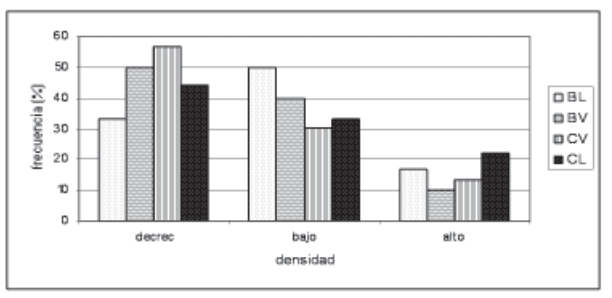

Figura 2: Comportamiento de la densidad de laurel a nivel de parcelas con una proyección a cinco años. 


\section{Análisis de escenarios}

\section{Sin aprovechamiento de individuos $\leq 45 \mathrm{~cm}$ de dap}

En este escenario, considerando la sobrevivencia del $100 \%$ de los individuos con dap $\leq 45 \mathrm{~cm}$ (Cuadro 10) se obtiene que en todos los sistemas agroforestales habrán incrementos en el ICAVc y el IPAvc, siendo los bananales los de mayores incrementos (13\% más que los cacaotales); sin embargo, los incrementos son bajos con respecto a la proyección con los datos observados.

Cuadro 5: Proyección de los incrementos volumétricos IPAvc de madera comercial de laurel por 5 años en cacaotales y bananales en valles y laderas sin aprovechamiento de dap $<45 \mathrm{~cm}$ en Talamanca, Costa Rica.

\begin{tabular}{lllllll}
\hline SAF & $\begin{array}{c}\text { Año 1 } \\
\text { ICAVc }\end{array}$ & $\begin{array}{c}\text { Año 2 } \\
\text { ICAVc }\end{array}$ & $\begin{array}{c}\text { Año 3 } \\
\text { ICAVc }\end{array}$ & $\begin{array}{c}\text { Año 4 } \\
\text { ICAVc }\end{array}$ & $\begin{array}{c}\text { Año 5 } \\
\text { ICAVc }\end{array}$ & IPAvc \\
\hline $\mathrm{CL}$ & 2,17 & 2,63 & 2,95 & 3,87 & 2,84 & 2,99 \\
\hline $\mathrm{CV}$ & 4,53 & 5,35 & 6,18 & 6.82 & 7,59 & 6,09 \\
\hline $\mathrm{BL}$ & 2,65 & 3,13 & 3,27 & 3,67 & 3,88 & 3,32 \\
\hline $\mathrm{BV}$ & 5,14 & 6,09 & 6,62 & 7,51 & 8,53 & 6,82 \\
\hline
\end{tabular}

CV: cacao en valle; CL: cacao en ladera; BV: banano valle; BL; banano ladera; SAF: sistemas agroforestales; ICAVc: incremento corriente anual en volumen comercial; IPAVc: incremento periódico anual del volumen comercial.

\section{Incrementos del $\mathbf{5 0 \%}$ de reclutamiento de individuos}

En este escenario, se obtiene un incremento en el volumen comercial de madera en todos los sistemas agroforestales evaluados siendo los bananales los que mayores incrementos presentan, no obstante los sistemas en laderas no presentan incrementos cuando se compararan con las proyecciones de los datos observados (Cuadro 11).

Cuadro 6: Proyección de los incrementos volumétricos IPAvc de madera comercial de laurel por cinco años en cacaotales y bananales en zonas de valles y laderas con incrementos del $50 \%$ de las tasas de reclutamiento en Talamanca, Costa Rica.

\begin{tabular}{ccccccc}
\hline \multirow{2}{*}{ SAF } & 2006 & 2007 & 2008 & 2009 & 2010 & \multirow{2}{*}{ IPAvc } \\
& ICAVc & ICAVc & ICAVc & ICAVc & ICAVc & \\
\hline
\end{tabular}




\section{SISTEMA AGROFORESTAL Y PRODUCCIÓN PECUARIA}

\begin{tabular}{lllllll}
\hline & $\left(\mathrm{m}^{3} \mathrm{ha}^{-1}\right)$ & $\left(\mathrm{m}^{3} \mathrm{ha}^{-1}\right)$ & $\left(\mathrm{m}^{3} \mathrm{ha}^{-1}\right)$ & $\left(\mathrm{m}^{3} \mathrm{ha}^{-1}\right)$ & $\left(\mathrm{m}^{3} \mathrm{ha}^{-1}\right)$ & $\left(\mathrm{m}^{3} \mathrm{ha}^{-1}\right)$ \\
\hline $\mathrm{CL}$ & 3,30 & 1,20 & 2,70 & 3,00 & 2,99 & 2,67 \\
\hline CV & 4,53 & 5,35 & 6,18 & 6,82 & 6,09 & 6,09 \\
\hline BL & 2,60 & 3,06 & 3,20 & 3,55 & 3,32 & 3,23 \\
\hline BV & 5,10 & 5,98 & 6,66 & 7,35 & 6,82 & 6,65 \\
\hline
\end{tabular}

CV: cacao en valle; CL: cacao en ladera; BV: banano valle; BL; banano ladera; SAF: sistemas agroforestales; ICAVc: incremento corriente anual en volumen comercial; IPAVc: incremento periódico anual del volumen comercial.

Los incrementos de madera tanto en cacaotales como en bananales son inferiores a los reportados en otros estudios realizados con laurel. Rosero y Gewald (1979) reportan incrementos en volumen de $14,8 \mathrm{~m}^{3} \mathrm{ha}^{-1}$ año-1 para la zona de Hone Creeck; Somarriba (1990) reporta incrementos en volumen de $9-24 \mathrm{~m}^{3} \mathrm{ha}^{-1} \mathrm{año}^{-1}$ para la zona de Turrialba. Sin embargo, los resultados obtenidos en este estudio son superiores a la proyección realizada por Suárez (2001) para estas mismas parcelas. Las bajas densidades poblacionales de laurel que manejan los agricultores de Talamanca determinan los bajos incrementos de volumen de madera, biomasa o carbono. Toda actividad de manejo que resulte en un incremento de la densidad permitirá mayores incrementos de volumen de madera.

En el análisis de los dos escenarios no se observan incrementos importantes en la producción de madera con respecto a los datos observados. Es probable que el período de análisis de cinco años es muy poco tiempo para que se exprese en el volumen de madera de laurel el efecto de mayores reclutamientos o el no aprovechamiento de individuos menores de $45 \mathrm{~cm}$ de dap. Con estas proyecciones, el sistema es sostenible porque hay incrementos de madera.

El modelo desarrollado puede considerarse como un reajuste al modelo de Suárez (2001), el mismo consideró el reclutamiento como una nueva variable, calculó la sobrevivencia a partir de dos momentos de evaluación (2001 y 2005) e incorporó datos de individuos desde las primeras clases diamétricas, por lo que el modelo gana en eficiencia. 


\section{Conclusiones}

- Los sistemas agroforestales de cacao y banano orgánico de la Reserva Indígenas de Talamanca son fuentes importantes de madera y almacenamiento de carbono.

- Los SAF de cacao en valle almacenan mayor volumen de madera que los cacaotales en ladera, bananales en valle y en laderas.

- En el período proyectado (2006-2010) con las matrices de transición, con las actuales tasas de extracción y reclutamiento, las poblaciones de laurel en los SAF aumentarán en (volumen y densidad).

- A nivel de parcelas individuales un $50 \%$ no tendrán incrementos y solamente un $15 \%$ presentaran incrementos altos.

- En los escenarios evaluados (disminución de la mortalidad e incrementos de las tasas de reclutamiento) los incrementos serán crecientes en todos los SAF.

\section{Recomendaciones}

- Se recomienda incidir en los agricultores indígenas para que incrementen las poblaciones de laurel en aquellas parcelas que presentan disminución de sus poblaciones a través de estimular la regeneración natural, un mejor manejo de las plantaciones de cacao y banano, así como un mejor aprovechamiento de los árboles que extraen.

- Se recomienda explorar la inserción de los sistemas agroforestales de la Reserva Indígena de Talamanca en el mercado del carbono como una forma de beneficio directo a los finqueros, la conservación de estos sistemas y estimular un mejor manejo de las plantaciones.

- Se recomienda el empleo de este modelo de proyección de los rendimientos e incrementos para aplicarlo como una herramienta de apoyo en la planificación y diseño de sistemas agroforestales con poblaciones de regeneración natural, disetaneas, en las que haya aprovechamiento y reclutamiento. 


\section{SISTEMA AGROFORESTAL Y PRODUCCIÓN PECUARIA}

\section{Lista de referencias}

Albertin, A; Nair, PK. (2004). Farmers` Perspectives on the role of shade Trees in Coffee Productions Systems: An assessment from the Nicoya Peninsula, Costa Rica. Human Ecology 32(4): 443-463.

Ávila, VG. (2000) Fijación y almacenamiento de Carbono en sistemas de café bajo sombra, a pleno sol, sistemas silvopastoriles y pasturas a pleno sol. Tesis Mag. Sc.CATIE, Turrialba, Costa Rica. 99 p.

Barrance, AJ; Flores, L; Padilla, E; Gordon, JE; Schreckenberg, K. (2003). Trees and farming in the dry zone of southern Honduras I: campesino tree husbandry practices. Agroforestry Systems 59: 97-106.

Beer, J. (1980). Cordia alliodora con Theobroma cacao: una combinación tradicional agroforestal en el trópico húmedo. CATIE. Turrialba, Costa Rica. 5p.

Boshier DH; Lamb AT. 1997. Cordia alliodora genética y mejoramiento de árboles. Oxford, UK, Oxford Forestry Institute. Tropical Forestry Papers No 36. 100 p.

Calvo, G; Meléndez L. (1999). Pseudoestacas de Laurel para el enriquecimiento de cacaotales. Agroforesteria en las Américas 6(22):25-27.

Duguma, B; Gockowski, J; Bakala, J. (2001). Smallholder Cacao (Theobroma cacao Linn.) cultivation in agroforestry systems of West and Central Africa: challenges and opportunities. Agroforestry Systems 51: 177-188.

Duguma, B; Gockowski, J; Bakala, J. (1999). Desafíos biofísicos y oportunidades para el cultivo sostenible de cacao (Theobroma cacao Linn.) en sistemas agroforestales de África Occidental y Central. Agroforestería en las Americas 6 (22): 12-15

Ford, L. (1979). An estimate of the yield of Cedrela odorata L. (Syn. Cedrela mexicana Roem.). UNU /CATIE WORKSHOP. Agro-forestry systems in tropical Latin America, Turrialba, Costa Rica. 11 p.

Guevara, MG. (1988). Experiencias Colombianas con cedro (Cedrela odorata L.). Convenio CONIF-Holanda. Serie documentación No 12. 85 p. 
Graves, A; Mac Carter, PS. (1990). Cordia alliodora a promising tree for topical agroforestry. Oxford, UK, Oxford Forestry Institute. Tropical Forestry Papers No 22. $37 \mathrm{p}$.

Kursten, E. (2000). Fuelwood production in agroforestry systems for sustainable land use and CO2 mitigation. Ecol Eng 16: S69-S72.

Marquez, L. (2000). Elementos Técnicos para inventarios de Carbono en uso del suelo. Fundación SOLAR. Guatemala. 31 p.

Nair, PK. (1997). Agroforestería. Centro de Agroforestería para el desarrollo sostenible. Universidad Autónoma de Chapingo, México. Editor L. Krishnamurthy. 543 p.

Pandey, DN. (2002). Carbon sequestration in agroforestry systems. Climate policy 2: $367-377$

Pezo D; Ibrahim, M. (1999). Sistemas Silvopastoriles. Proyecto Agroforestal CATIE/ GTZ. (Materiales de Enseñanza CATIE No. 44). CATIE, Turrialba, Costa Rica. $276 \mathrm{p}$.

Roshetko, J; Delaney, M; Hairiah, K; Purnomosidhi P. (2002). Carbon stocks in Indonesia homegarden systems: Can smallholder systems be targeted for increased carbon storage?. America Journal of Alternative Agriculture 17(2): 138-148.

Somarriba, E; Trivelato, M; Villalobos, M; Suárez, A; Benavides, P; Morán, K; Orozco, L; López, A. (2003). Diagnóstico agroforestal de pequeñas finca cacaoteras orgánicas de Indígenas Bribri y Cábecar de Talamanca, Costa Rica. Agroforestería en las Américas. 10(37-38):24-30.

Somarriba, E. (1994). Maderables como alternativa para la sustitución de sombra de cacaotales establecidos. El concepto. Turrialba, Costa Rica, CATIE 34 p. (Serie Técnica. Informe Técnico No. 238).

Suárez, A. (2001). Aprovechamiento sostenible de madera de Cordia alliodora y Cedrela odorata de regeneración natural en cacaotales y bananales de indígenas de Talamanca, Costa Rica. Tesis Mag. Sc. Turrialba, Costa Rica, CATIE. 77 p.

Suárez, D. (2002). Cuantificación y valoración económica del servicio ambiental y almacenamiento de carbono en sistemas agroforestales de café en la comarca 


\section{SISTEMA AGROFORESTAL Y PRODUCCIÓN PECUARIA}

Yassica Sur, Matagalpa, Nicaragua. Tesis Mag. Sc. Turrialba, Costa Rica, CATIE. $131 \mathrm{p}$.

Sharrow, SH; Ismail, S. (2004). Carbon and Nitrogen storage in agroforests, tree plantations, and pastures in western Oregon, USA. Agroforestry Systems 60: 13:130.

Tufekcioglu, A; Raich, JW; Isenarth, TM; Schultz, RC. (2003). Biomass, carbon and nitrogen dynamics of multi-species riparian buffers within an agricultural watershed in Iowa, USA. Agroforestry Systems. 57: 187-198.

Vanklay, J. (1994). Modelling forest growth and yield Applications to Mixed Tropical Forests. Cab International, Oxford, UK. 312 p.

Vandermeer, J. (1981). Elementary mathematical ecology. John Wiley and Sons. New York. 291 p. 